\section{On Abstract Gronwall Lemmas}

\section{N. Lungu*}

Professor, Technical University of Cluj-Napoca, Romania

In this paper, we present some problems related to Abstract Gronwall Lemmas. We begin our considerations with some notions from operatorial inequality [1-6].

Let $(X, \rightarrow, \leq)$ be an ordered L-space [2-6], $A: X \rightarrow X$ an operator. We denote $\mathrm{F}_{\mathrm{A}}$ as the fixed points set of $\mathrm{A}$. We consider that the equation $\mathrm{x}=\mathrm{A}(\mathrm{x})$ has a unique solution $x_{A}^{*}$. The operatorial inequality problem is the following [1-4]:

Find conditions under which

(i) $x \leq A(x) \Rightarrow x \leq x_{A}^{*}$;

(ii) $x \geq A(x) \Rightarrow x \geq x_{A}^{*}$.

To have a concrete result for this problem it is necessary to determine the $x_{A}^{*}$, or to find $\mathrm{y}: z \in X$ such that $x_{A}^{*} \in[y, z]$.

Abstract Gronwall Lemma (AGL) $[2,3,5,6]$.

Let $(X, \rightarrow, \leq)$ be an ordered L-space and $A: X \rightarrow X$ an operator.

We suppose that

(i) A is PO;

(ii) $\mathrm{A}$ is increasing.

Then,

(i) $x \leq A(x) \Rightarrow x \leq x_{A}^{*}$;

(ii) $x \geq A(x) \Rightarrow x \geq x_{A}^{*}$.

Abstract Gronwall-Comparison Lemma (AGCL) [2,3,5,6]. Let $(X, \rightarrow, \leq)$ be an ordered L-space and $A, B: X \rightarrow X$ two operators. We suppose that:

(i) $\mathrm{A}$ and $\mathrm{B}$ are POs;

(ii) A is increasing;

(iii) $A \leq B$.

Then,

$$
x \leq A(x) \Rightarrow x \leq x_{B}^{*} .
$$

We present two problems as follows. First is the simplest and most useful integral inequality.

Lemma Gronwall [7-9]. Let $y, g \in C\left(\left[a, b, \mathbb{R}_{+}\right)\right.$

Suppose, for $c \geq 0$, we have

$$
y(x) \mid \leq c+\int_{a}^{x} y(s) g(s) d s, x \geq a \geq 0 .
$$

Then,

$$
y \leq c \exp \left(\int_{a}^{x} g(s) d s\right), x \geq a \geq 0
$$

In this case $(X, \stackrel{\|\|}{\longrightarrow}, \leq)=C([a, b], \stackrel{\|\|}{\longrightarrow} \leq)$, where $\|$.$\| is the Bielecki norm$ on $C([a, b]),\|y\|:=\max _{x \in[a, b]}\left(|y(x)| e^{-T(x-a)}\right), T \in \mathbb{R}_{+}^{*}$ and $A: X \rightarrow X$ is defined by

$$
A(y)(x)=c+\int_{a}^{x} y(s) g(s) d s
$$

The fixed point of the operator A is $x_{A}^{*}=c \exp \left(\int^{x} g(s) d s\right)$ and the inequality (3) derived from AGL. have:

The second problem is analogous to Gronwall lemma. Thus, we

Lemma Wendorff [1,7-9]. We consider that

(i) $u, v \in C\left([0, a] \times[0, b], \mathbb{R}_{+}\right), c \in \mathbb{R}_{+}$;

(ii) $v$ is increasing

If $\mathrm{u}$ is a solution of inequality

$$
u(x, y) \leq c+\int_{0}^{x} \int_{0}^{y} v(s, t) u(s, t) d s d t, x[0, a], y \in[0, b]
$$

then

$$
u(x, y) \leq c \exp \left(\int_{0}^{x} \int_{0}^{y} v(s, t) d s d t\right)
$$

In this case, the operator $\mathrm{A}$ is the second part of (5) inequality, but the function $u(x, y) \leq \exp \left(\int_{0}^{x} \int_{0}^{y} v(s, t) d s d t\right)$ is not the fixed point of the operator A. This inequality is derived from AGCL.

For other applications see Lungu and Popa [10], Lungu and Rus [11], and Popa and Lungu [12].

\section{References}

1. Craciun C, Lungu N (2009) Abstract and Concrete Gronwall Lemmas. Fixed Point Theory 10: 221-228.

2. Lungu N, Rus IA Gronwall inequalities via Picard operators (to appear).

3. Rus IA Gronwall Lemmas: Ten Open Problems. Scientiae Math Japonicae.

4. Rus IA (2004) Fixed points, upper and lower fixed points: abstract Gronwall lemmas. Carpathian J Math 20: 125-134.

5. Rus IA (2003) Picard operators and applications. Scientiae Math Japonicae 58: 191-219.

6. Rus IA (1993) Weakly Picard mappings. Comment Math Univ Carolin 34: 769773.

*Corresponding author: N. Lungu, Professor, Technical University of Cluj-Napoca, Romania, E-mail: nlungu@mail.utcluj.ro

Received August 07, 2012; Accepted August 11, 2012; Published August 16 , 2012

Citation: Lungu N (2012) On Abstract Gronwall Lemmas. J Applied Computat Mathemat 1:e119. doi:10.4172/2168-9679.1000e119

Copyright: (c) 2012 Lungu N. This is an open-access article distributed under the terms of the Creative Commons Attribution License, which permits unrestricted use, distribution, and reproduction in any medium, provided the original author and source are credited. 
7. Bainov D, Simeonov P (1992) Integral Inequalities and Applications. Kluwer Academic Publishers, The Netherlands.

8. Lakshmikantham (1988) Stability Analysis of Nonlinear Systems. Taylor \& Francis.

9. Pachpatte BG (1998) Inequalities for Differential and Integral Equations: Academic Press, San Diego, CA.
10. Lungu N, Popa D (2002) On some differential inequalities. Seminar of Fixed Point Theory 3: 323-327.

11. Lungu N, Rus IA (2001) Hyperbolic differential inequalities. Libertas Mathematica 21: 35-40.

12. Popa D, Lungu N (2005) On an operatorial inequality. Demonstratio Mathematica 38: 667-674. 